\title{
Antimicrobial Lessons From a Large Observational Cohort on Intra-Abdominal Infections in Intensive Care Units
}

Dirk Vogelaers ${ }^{1,2 *}$ (ORCID: 0000-0002-7707-285X),

Stijn Blot ${ }^{*}$ (ORCID: 0000-0003-2145-0345),

Andries Van den Berge ${ }^{1}$ (ORCID: 0000-0002-8630-5651),

Philippe Montravers ${ }^{3}$ (ORCID: 0000-0002-3422-5705), for the Abdominal Sepsis Study (“AbSeS") group on behalf of the Trials Group of the European Society of Intensive Care Medicine**

${ }^{1}$ Department of Internal Medicine and Pediatrics, Faculty of Medicine and Health Sciences, Ghent University, Belgium

${ }^{2}$ Department of General Internal Medicine and Infectious Diseases, AZ Delta, Deltalaan 1, 8800 Roeselare, Belgium

${ }^{3}$ Université de Paris, UFR Denis Diderot - INSERM UMR 1152, ANR-10-LABX-17 - Department of Anaesthesiology and Critical Care Medicine, DMU PARABOL, CHU Bichat-Claude Bernard, AP-HP, Paris, France

* Joint first authorship

**AbSeS national coordinators and local investigators are mentioned in ESM-1

Word count: 4620

Address of correspondence:

Prof. Dr. Stijn BLOT

Dept. of Internal Medicine and Pediatrics

Faculty of Medicine \& Health Sciences

Ghent University

Campus UZ Gent

Corneel Heymanslaan 10

9000 Ghent, Belgium 


\begin{abstract}
(word count: 250)
\end{abstract}
Severe intra-abdominal infection commonly requires intensive care. Mortality is high and mainly determined by disease-specific characteristics, i.e. setting of infection onset, anatomical barrier disruption, and severity of disease expression. Recent observations revealed that antimicrobial resistance appears equally common in community-acquired and late-onset hospital-acquired infection. This challenges basic principles in anti-infective therapy guidelines, including the paradigm that pathogens involved in community-acquired infection are covered by standard empiric antimicrobial regimens, and secondly, the concept of nosocomial acquisition as main driver for resistance involvement. In this study we report resistance profiles of Escherichia coli, Klebsiella pneumoniae, Pseudomonas aeruginosa, Enterococcus faecalis and Enterococcus faecium in distinct European geographic regions based on an observational cohort study on intra-abdominal infections in ICU patients. Resistance against aminopenicillins, fluoroquinolones, and $3^{\text {rd }}$ generation cephalosporins in E. coli, K. pneumoniae and P. aeruginosa is problematic as is carbapenemresistance in the latter pathogen. For E. coli and $K$. pneumoniae resistance is mainly an issue in Central Europe, Eastern \& South-East Europe, and Southern Europe, while resistance in $P$. aeruginosa is additionally problematic in Western Europe. Vancomycin-resistance in E. faecalis is of lesser concern but requires vigilance in E. faecium in Central and Eastern \& South-East Europe. In the subcohort of patients with secondary peritonitis presenting with either sepsis or septic shock, appropriateness of empiric antimicrobial therapy was not associated with mortality. In contrast, failure of source control was strongly associated with mortality. The relevance of these new insights for future recommendations regarding empiric antimicrobial therapy in intra-abdominal infections is discussed.

\title{
Key points.
}

- Recent observations revealed that antimicrobial resistance appears equally common in community-acquired and late-onset hospital-acquired intra-abdominal infection. In this study we found that resistance against aminopenicillins, fluoroquinolones, and $3^{\text {rd }}$ generation cephalosporins in E. coli, K. pneumoniae and P. aeruginosa is problematic as is carbapenemresistance in the latter pathogen.

- In addition, we observed that in critically ill patients with secondary peritonitis appropriateness of antimicrobial therapy was not associated with a survival benefit. In the context of intensive care, source control appears much more important determinant of mortality.

- These insights incite reconsideration of current recommendations of anti-infective therapy 
with emphasis on defining resistance thresholds, and a selection of empiric antimicrobial therapy based on local microbial resistance patterns, individual colonization status, severity of disease, PK/PD optimization and duration of antimicrobial therapy.

\section{Short title.}

Antimicrobial resistance in intra-abdominal infections

\section{Keywords.}

intra-abdominal infection; peritonitis; multidrug resistance; antimicrobial resistance; antibiotic resistance; outcome; mortality; antimicrobial therapy; antibiotic therapy; source control; intensive care

\section{Declarations.}

Funding. AbSeS is a Trials Group Study of the European Society of Intensive Care Medicine. The study was supported by a Pfizer investigator-initiated research grant.

Conflicts of interest. SB received research grants from Pfizer. PM served as speaker and advisory board member for Menarini, MSD and Pfizer. DV and AVdB have no conflicts of interest to disclose.

Availability of data and materials. Due to the numerous data user agreements in this multicenter study, acess to the data is exclusively grated to the ESICM and the head investigator at Ghent University (SB).

Ethics approval. Approval by established national, regional, or local institutional review boards was expedited and granted. The study is registered at ClinicalTrials.gov (number NCT03270345).

Consent. AbSeS is an observational study. Necessity of written informed consent was determined by the national, regional, or local ethics committee or institutional review board.

\section{Author contributions.}

DV contributed to the conception and design of the study, interpretation of the results, drafting some parts of first version of the manuscript, and revision of the full paper for important intellectual content.

SB contributed to the conception and design of the study, data management, data analysis and interpretation, drafting some parts of first version of the manuscript, and revision of the full paper for important intellectual content.

$\mathrm{AVdB}$ contributed to data management, data analysis and interpretation, and revision of the paper for important intellectual content.

PM contributed to the conception and design of the study, data acquisition, interpretation of the results, and revision of the paper for important intellectual content.

All authors read and approved the final manuscript. 


\section{Introduction}

The denominator of intra-abdominal infection (IAI) covers a large spectrum of disease, that either involves a single organ without anatomical disruption or an increasing degree of complexity ranging from localized peritonitis over intra-abdominal abscedation to diffuse peritonitis, with a range of disease severity up to septic shock.[1] Hence caution is warranted in lumping this spectrum together into a single category, as this carries risks of generalization and diagnostic reductionism.[2]

IAI represents an important cause of morbidity and mortality, especially with severity of disease expression requiring intensive care unit (ICU) admission, or when IAI complicates the course of already critically ill patients.[3,4] In the latter, mortality is determined mainly by (i) setting of infection acquisition (either community-acquired, healthcare-associated or early-onset hospitalacquired, and late-onset hospital-acquired), (ii) the presence of anatomical barrier disruption (i.e. no disruption, or either disruption with localized or diffuse peritonitis), and (iii) severity of disease expression (i.e., either infection, sepsis or septic shock) (Table 1).[2,5] A grid of these three major disease characteristics results in the definition of 27 possible phenotypes. They have been linked with outcome as demonstrated in a large multinational, multicenter cohort study of 2621 critically ill patients with an overall mortality of 29.1\%.[5] However mortality ranged widely within this spectrum of phenotypes. This grim prognosis can be explained by the essential role of adequate source control that may be a particular challenge in complicated cases on one hand, and the wide spectrum of potential pathogenic micro-organisms involved. Besides the broad range of potential pathogens, the global emergence of steadily increasing antimicrobial resistance is of great concern as it may lead to inappropriate empiric therapy potentially further burdening outcome.[6] This adequacy of anti-infective therapy is judged to play a determinant role in the prognosis of IAI.[7,8] However, a number of recent studies have failed to demonstrate the importance of adequate empirical antibiotic therapy in IAI.[9-12] This constitutes a dilemma for clinicians as they face increasing emergence of resistance together with an abundance of studies demonstrating the deleterious impact of resistance and inappropriate antibiotic therapy in IAI as in other infections.[8,13-17]

The objective of this narrative review is to discuss the current status of antimicrobial resistance within the context of IAI in critically ill patients, introducing new original research data on resistance patterns from the AbSeS study across different European geographic regions. To assess the relative importance of antimicrobial therapy, the associations with mortality will be evaluated in a selection of patients with secondary peritonitis for appropriateness of empiric antimicrobial therapy on one hand, and source control achievement on the other hand. The impact of antimicrobial resistance on the outcome of IAI requiring intensive care support will be critically assessed, leading to 
recommendations towards guidelines of management of truly complicated IAI.

\section{Methods}

A narrative review on IAI was conducted on the basis of recent literature, most of which published in the past decade. To support the discussion, we performed secondary analyses of data from the AbSeS study.[5] This observational, prospective, international cohort included critically ill adult patients with IAI from 309 ICUs and 42 countries between January and December 2016. Approval by established national, regional, or local institutional review boards was expedited and granted. The study is registered at ClinicalTrials.gov (number NCT03270345). Protocols and procedures for the patients' inclusion criteria, definitions, methods and collection of data, have been previously reported.[5] Microbiological assessment was left at the discretion of the physician. Eligible cultures included intra-operative cultures, cultures from trans-abdominal fine-needle aspiration and from abdominal drains sampled $\leq 24 \mathrm{~h}$ post-surgery, as well as blood cultures with isolation of pathogens likely to be related to the IAI. However, blood cultures results were left out of the present analysis because of the relative uncertainty that they were truly originating from the IAI. The thresholds for resistance reported by The European Committee on Antimicrobial Susceptibility Testing (EUCAST) were used.[18] Both microbiological assessment and susceptibility testing (i.e., the spectrum of agents tested) were executed according to local laboratory routine procedures. As a consequence there is variability in the anti-infective agents tested.

For the present analyses on antimicrobial resistance patterns, only data from patients admitted to European ICUs were included. Our aim was to describe the patterns in antimicrobial resistance across four European regions, i.e. Western-Europe (UK, France, Belgium, the Netherlands), CentralEurope (Czech Republic, Switzerland, Germany, Poland, Denmark), Eastern- \& South-Eastern Europe (Serbia, Croatia, Romania, Russian Federation), and Southern-Europe (Greece, Italy, Portugal, Spain). In addition, we decided to only consider microorganisms for which $>100$ strains were available in the AbSeS database for the European region. These included Escherichia coli, Klebsiella pneumoniae, Enterococcus faecalis, Enterococcus faecium, and Pseudomonas aeruginosa. We compared the data of these five micro-organisms with general European surveillance data as reported by the European Centre of Disease Prevention and Control (ECDC).[19] For this purpose, antibiotics were clustered in main classes of clinical importance, i.e. for E. coli: aminopenicillins (without beta-lactam inhibitor), fluoroquinolones, $3^{\text {rd }}$ generation cephalosporins, aminoglycosides, and carbapenems; for $K$. pneumoniae: fluoroquinolones, $3^{\text {rd }}$ generation cephalosporins, aminoglycosides, and carbapenems; for P. aeruginosa: piperacillin/tazobactam, fluoroquinolones, ceftazidime, aminoglycosides, and carbapenems; for E. faecalis and E. faecium: aminopenicillins 
(without beta-lactam inhibitor) and vancomycin. According to ECDC reporting, a strain was considered 'resistant' against an antibiotic class if resistant against at least one of the tested antibiotics within that class. Because of the clinical relevance of some antibiotic agents in the context of IAIs, such as ampicillin/sulbactam, amoxicillin/clavulanate, piperacillin/tazobactam, tigecycline, and colistin, we additionally reported all available resistance data from the AbSeS study for the distinct European geographic regions (ESM-2).

In a second step, we assessed the relative importance of antimicrobial therapy by analyzing data from patients with secondary peritonitis with either sepsis or septic shock within the AbSeS database, through an analysis of the relationship between appropriateness of empiric antimicrobial therapy and mortality. Empiric antimicrobial therapy was considered appropriate if the regimen initiated within the first 24 hours of IAI onset included an antimicrobial agent with in vitro and likely clinical activity against the isolated pathogens. Only patients with positive culture results were considered. In addition, on the same set of patients we assessed the relationship between achievement of source control and mortality. Source control achievement was evaluated at day 7 or earlier if the patient died within that timeframe. Source control was judged as either successful or having failed. Failure was defined as either persistent inflammation (clinical evidence of a remaining intra-abdominal source of infection) or the necessity of surgical revision. Mortality was evaluated at the end of the ICU stay, with a minimum of 28 days of observation. Mortality associated with either appropriateness of empiric antimicrobial therapy and source control achievement was reported according to a grid of other likely drivers of outcomes in patients with IAI: (i) setting of IAI onset (community-acquired, early- or late-onset hospital-acquired), (ii) localized or diffuse peritonitis, and (iii) severity of disease expression, either sepsis or septic shock. Concerning the latter, patients with 'infection' (i.e. without sepsis or septic shock) were left out of the analysis because of their smaller number and limited impact on mortality.[5] The Chi-square test was used for all analysis as appropriate ( $\mathrm{n} \geq 5$ in all cells required).

\section{Microbiology in intra-abdominal infections}

The gastrointestinal tract, in particular the terminal ileum and colon, is characterized by a microbiome consisting of hundreds of species, mainly with low pathogenic potential keeping the balance in the interbacterial competition for colonization, thereby hampering true pathogens to become dominant species, coined as colonization resistance. This colonization with potentially pathogenic microorganisms can be a starting point for nosocomial infections, through translocation or cross-infection.[20] IAI themselves can be caused by a broad range of pathogens, mainly originating from the endogenous flora containing Gram-negatives such as Enterobacterales with 
Escherichia coli as dominant species, Gram-positives such as streptococci and enterococci, and anaerobic bacteria. In patients with a disturbed colonization resistance due to critical illness or antimicrobial exposure, transient micro-organisms including Staphylococcus aureus, Pseudomonas aeruginosa, and Candida species can establish themselves in the gastrointestinal tract and need to be considered as well. As a rule, IAI are polymicrobial, involving a Gram-negative moiety responsible for the septic inflammatory response and anaerobes, responsible for abscess formation, a so-called two-stage disease concept.

\section{The problem of antimicrobial resistance in intra-abdominal infections in critically ill patients}

Antimicrobial resistance is a global, continuously expanding problem defined as a top-10 global public health threat by the World Health Organization. Of particular concern are methicillinresistance in S. aureus, vancomycin-resistance in E. faecium, and carbapenem-resistance and extended-spectrum beta-lactamase (ESBL) production in Gram-negatives with Acinetobacter baumannii, K. pneumoniae, and P. aeruginosa as key pathogens.[21] In the context of IAI however, S. aureus and A. baumannii are of minor relevance given their low frequency of involvement.[5,8,22] In general, the risk of involvement of resistant pathogens is perceived to be low in communityacquired infections, moderate in early-onset hospital-acquired infection, and high in late-onset hospital-acquired infection, especially when occurring in critically ill patients. Three decades ago, the intensive care unit (ICU) could still be considered the epicenter of antimicrobial resistance. However, over time, the resistance problem has spread into the entire hospital, nursing homes and other healthcare facilities, and the broader community. Illustrative for expanding endemicity of antimicrobial resistance is the fact that classic risk factors for resistance have lost predictive value. In the 1990s prolonged hospitalization and/or recent antibiotic exposure effectively predicted the likelihood of antimicrobial resistant pathogens in hospital-acquired infections in critically ill patients.[23,24] In a study exploring risk factors for antimicrobial resistance in a cohort of patients with post-operative peritonitis, the initiation of antibiotic therapy in addition to standard perioperative coverage appeared the only significant predictor for resistance involvement.[25] In patients with antimicrobial resistant bacteria, the average time to surgical revision was 13 days and not significantly longer compared with patients with infection due to other bacteria (11 days on average).[25] In a Belgian multicenter study conducted in 2006-2007, nearly 40\% of nosocomial infections in critically ill patients, occurring in the absence of classic risk factors (i.e., recent antibiotic exposure and length of hospitalization $>7$ days), were caused by resistant pathogens.[26] More recent data further supported the concept that the distinction between community-acquired $v s$. hospital-acquired infection onset no longer seems associated with a clear-cut distinction between low 
vs. high risk for AMR involvement. In the AbSeS study, prevalence of antimicrobial resistance in clinically significant samples was not different in community-acquired (26.5\%) as compared to earlyonset $(29.0 \%)$ and late-onset hospital acquired intra-abdominal infections $(24.6 \%)(\mathrm{p}=0.215)$ and this observation was valid for the total cohort as for distinct geographic regions.[5] Besides antimicrobial resistance, no pathogen proved particularly dominant in either community-acquired, early-onset or late-onset hospital-acquired IAI, thereby undermining the 'classic' presumption that $P$. aeruginosa, and E. faecium, not covered by standard first-line antimicrobial therapy but feared for their resistance potential, are particularly important in the latter group, at least in the context of critical illness.[2732] Although the prevalence of $P$. aeruginosa is relatively low (3\% to $11 \%$ ), even in the context of late-onset peritonitis $[5,8,33]$, this pathogen is given substantial attention because of its high pathogenicity once it causes invasive disease. The relative importance of $P$. aeruginosa in late-onset IAI is illustrated by Montravers et al. in a study exploring shifts in microbiology in patients undergoing up to three surgical revisions following persistent peritonitis.[33] Occurrence of $P$. aeruginosa increased from $4 \%$ in the initial surgery to $11 \%$ in the third revision but this difference was not statistically significant. First-line empiric coverage of enterococci is also not strictly recommended but their coverage is generally advocated in patients with an at risk profile including either septic shock, prolonged exposure to cephalosporins, immunosuppressed status, prosthetic heart valves, and recurrent peritonitis.[34-36] Regarding the latter, Montravers et al. found E. faecalis involvement to be stable in patients undergoing subsequent surgical revisions for peritonitis (approximately 6\%), while E. faecium involvement showed a non-significant increase from $4 \%$ in the initial surgery to $9 \%$ in a third reoperation. The observation that multidrug resistance appears equally important in community-acquired as in late-onset hospital infection, and that neither $P$. aeruginosa nor E. faecium appears more prevalent in recurrent peritonitis challenges some basic principles in the development of guidelines in empirical antimicrobial therapy. On one hand, it defies the assumption that pathogens are presumed to be susceptible to first-line antibiotics in community-acquired infections. On the other hand, it questions the principle that nosocomial acquisition represents a major driver for multidrug resistance involvement, due to the selective pressure of prior exposure to antimicrobials and patient colonization with resistant pathogens in the care setting. This opens the discussion on thresholds of resistance to key antimicrobials requiring rethinking of empiric regimens. In general, these thresholds will largely depend on severity of disease expression and forgiveness of inappropriateness of initial therapy. This is illustrated by a practically zero tolerance for even low levels of clinically significant resistance in key pathogens for acute bacterial meningitis caused by meningococci and pneumococci in the choice of empiric antibiotic regimen versus acceptable thresholds for resistance in urinary tract infections (UTI). In lower UTI, mainly cystitis, a resistance 
threshold of $20 \%$ of clinically relevant isolates in a specific setting is accepted, vs. $10 \%$ in upper UTI, mainly pyelonephritis.[37-39] Actually, in community-acquired E. coli pyelonephritis without septic shock, fluoroquinolone-resistance do not impact outcome in patients with initial empiric fluoroquinolone therapy.[40]

This does not necessarily imply that this increased spread of resistance through hospital and community is due to the ICU context itself, with more debilitated and severely ill patients exposed to prior antimicrobials and risk for cross-infection in intensive care, as also in severe communityacquired IAI a high prevalence of antimicrobial resistance is observed. This is probably due to the erosion of the sharp interface between community-acquired and nosocomial infection, due to escape of resistance mechanisms from the hospital into the community and the spread of resistance mechanisms in the community itself. This is illustrated by the high incidence of e.g. ESBL-producing or carbapenem-resistant Enterobacterales in travelers returning from South-East Asia, of ESBLgenes in the food chain.[41-47] Furthermore, in a three-national cohort of 3600 patients planned for elective colorectal surgery, pre-operative rectal swabs showed a large variability of bowel carriage of ESBL-producing Enterobacterales, reaching 28.8\% in Israel, 12.0\% in Switzerland, and 9.4\% in Serbia.[20] Insufficiently controlled use of antimicrobials in the community may promote spread of resistance genes. In the AbSeS study, patients with antimicrobial exposure in the 6 months preceding the episode of IAI were classified together with early-onset hospital-acquired infection. This runs against the classic definitions of nosocomial infection, restricted to the hospitalization period excluding manifestation of infection within the first two days after admission and the 30 days following hospital discharge. Ever increasing quality of medical care and therapeutic options resulting in improved survival rates for a wide spectrum of disease leads to a higher comorbidity index in the community; hence, a considerable proportion of patients with community-acquired IAI had at least one type of underlying disease, associated with a higher likelihood of prior recent antimicrobial exposure, whatever cutoff is used $(<6$ or $<3$ months prior to the actual hospital admission for severe community acquired IAI).

\section{Regional differences in antimicrobial resistance in intra-abdominal infection}

Pooled antimicrobial resistance data (i.e. either MRSA, VRE, or ESBL-producing, carbapenemresistant or fluoroquinolone-resistant Gram-negatives) as reported in $\mathrm{AbSeS}$ were $26.3 \%$ on average.[5] However, resistance rates differed substantially between regions, with West-Europe having the lowest rates $(10.5 \%)$ in contrast to worrisome rates in Latin-America (38.6\%), Eastern and South-East Europe (43.0\%), and North Africa \& Middle-East (50.6\%). 
In this study, we report resistance data on 772 strains of E. coli, 227 strains of K. pneumoniae, 129 strains of P. aeruginosa, 232 strains of E. faecalis, and 168 strains of E. faecium. As the study collected strains isolated through three different sampling methods, data from 2 strains of the same microorganism sampled from a single patient may have been reported. However, as the gastrointestinal tract may contain different strains from the same species, we did consider all cultures isolated.

Tables 2 to 6 detail antimicrobial resistance rates for clinically important pathogens in IAIs as observed in four different European geographic regions. The data must be interpreted cautiously as the number of tested strains can be low for key pathogens in a particular region. In summary, in $E$. coli (Table 2) fluoroquinolone-resistance is significantly higher in Central Europe, Eastern \& SouthEast Europe, and Southern Europe $(27.7 \%, 20.9 \%, 23.1 \%$, respectively) compared with Western Europe (12.5\%). Carbapenem-resistance is only an issue in Eastern \& South-East Europe (15.9\%) and resistance against aminoglycosides is relevant in Central European countries (14.0\%). For $K$. pneumoniae (Table 3), resistance rates for all relevant antibiotic classes were higher in Central Europe, Eastern \& South-East Europe, and Southern Europe compared with Western Europe. Resistance in P. aeruginosa (Table 4) is an issue in all European regions. For all antibiotic classes, resistance rates frequently exceed the $20 \%$ threshold, also in Western Europe. Exceptionally high resistance rates are observed in Southern Europe against piperacillin/tazobactam (37.5\%) and carbapenems (38.3\%), and in Eastern \& South-East Europe against fluoroquinolones (35.5\%), aminoglycosides (38.5\%), and carbapenems (45.0\%). Of note, also in Western Europe carbapenemresistance in P. aeruginosa approaches the 30\% threshold. Aminopenicillin-resistance in E. faecalis (Table 5) is a particular issue in Eastern \& South-East Europe (30.6\%), while VRE is in general of lesser concern. In E. faecium (Table 6) strains, aminopenicillin-resistance is high in all European regions (>70\%). The prevalence of VRE appears higher in Central (15.4\%) and Eastern \& South-East Europe (15.2\%), but numbers of isolates are low.

The high resistance levels for aminopenicillins, 3rd generation cephalosporins and, to a lesser extent, for fluoroquinolones in E. coli as in K. pneumoniae, seemingly favor carbapenems as first-line empiric choice. The trend towards empiric carbapenem use has been recently illustrated in a multicenter cohort of 4453 patients in the US treated for community-acquired (62\%), healthcareassociated (27\%), and hospital-acquired complicated IAI (10\%).[48] An empirical carbapenem regimen was given in $24 \%$ of patients with community-acquired IAI, $29 \%$ of patients with healthcare-associated IAI and $35 \%$ of patients with hospital-acquired IAI. In this regard, it is however important to realize that data from individual hospitals may substantially deviate from the national average, in community-acquired as well as in hospital-acquired infections, indicating that 
considerations regarding empiric therapy must be based on local epidemiology.[49] Besides local epidemiology, we suggest to use carbapenems on basis of disease severity because in patients with septic shock there is little room for missing the causative pathogens. The relative contribution of appropriate empiric antibiotic therapy compared with source control underscores the need for a restrictive policy towards carbapenem use in patients with minor disease severity. This requires a shift in mindset as aiming for a high rate of appropriate empiric therapy has been the mantra for the past two decades and, evidently, upfront use of carbapenems implies a higher likelihood of appropriateness of therapy.[25,26]

To allow direct comparison with general resistance data in the respective geographic regions, Tables 2 to 6 also report percentages of resistance provided by the ECDC for blood isolates of the same pathogen. Although some striking differences can be observed (for example, in Southern Europe, for fluoroquinolone-resistance in E. coli [Table 2] or carbapenem-resistance in K. pneumoniae [Table 3]), a distinct pattern cannot be discerned. This can be explained by relatively smaller numbers of isolates in the AbSeS study compared with the European-wide surveillance system. Furthermore, the limited small number of participating ICUs may reflect local resistance patterns rather than national average data. In any case, the often high and geographically variable resistance rates in IAI and the observed differences between IAI vs. ECDC's blood isolates stress the importance of steering antimicrobial therapy on local susceptibility data.

To the best of our knowledge, no prior study has compared ESBL isolation rates in IAI versus other clinical isolates. It remains uncertain to which extent ESBL fecal carriage represents an exclusive risk factor for infectious complications following abdominal surgery. In the study by DubinskyPertzov et al. carriage of ESBL-producing Enterobacterales appeared an independent risk factor for surgical site infection (odds ratio [OR] 2.36, 95\% confidence interval [CI] 1.50-3.71) and deep incisional or organ/space infection (OR 2.25, 95\% CI 1.27-3.99).[20] Furthermore, Golzarri et al. found ESBL-producing Enterobacterales to be an independent risk factor for both surgical site infection (relative risk [RR] 2.16, 95\% CI 1.20-3.90) and for bloodstream infection (RR 4.02, 95\% CI 2.36-6.87) in a cohort of cancer patients undergoing gastrointestinal or gynecologic surgery.[50]

\section{Appropriateness of empiric antimicrobial therapy, source control, and mortality in critically ill patients with secondary peritonitis}

From the AbSeS database, 1002 critically ill patients could be retrieved with secondary peritonitis, either sepsis or septic shock, and with data reported on culture results, antimicrobial therapy, and source control. The overall rate of empiric appropriate antimicrobial therapy was $64.8 \%$. The 
categories of localized and diffuse peritonitis within community-acquired, early- and late- hospitalacquired onset of IAI in the AbSeS study with sepsis or septic shock as severity of disease index were further analyzed towards the effects of appropriateness of initial empiric antimicrobial therapy, and reported effectiveness of initial source control towards mortality. In the context of secondary peritonitis associated with sepsis or septic shock guidelines usually recommend pseudomonal and enterococcal coverage in addition to the basic scheme targeting Gram-positive, Gram-negative, and anaerobic bacteria.[51] Such a broad coverage resulted in a significantly higher rate of appropriate empiric antibiotic coverage albeit still with room for improvement indicating that specific resistance patterns are responsible for the inappropriateness of antibiotic therapy $(74.7 \%$ vs. $45.9 \%$; $<<0.001)$. No significant difference in mortality could be documented between patients with appropriate $v s$. inappropriate empiric antimicrobial therapy (Table 7), nor with pooling of all patients with sepsis (24.7\% vs. $29.0 \% ; \mathrm{p}=0.211)$ or septic shock separately $(37.9 \%$ vs. $45.7 \% ; \mathrm{p}=0.183)$ or combined (28.1\% vs. $34.0 \%$; $=0.099)$. The latter non-significance must be put in perspective. Patients who received appropriate antibiotic therapy had a survival benefit of $6 \%$. For this observed difference in mortality to become statistically significant a larger sample size of 1936 patients, actually double that available within the AbSeS study, would have been required on the basis of a post hoc power analysis. This could represent a feasible dedicated research project, preferably focusing on patients with adequate primary source control, in order to avoid major confounders. Rates of appropriate empiric antibiotic therapy in IAI range $55 \%$ to $91 \%$ but differences in settings, case-mix, and microbial etiology hamper direct comparisons.[12,52-54] Yet, few investigators reported on rates of empiric appropriate therapy in IAIs probably because of the equivocal meaning of perioperative culture results in secondary peritonitis.

In contrast with appropriateness of empiric antibiotic therapy, failure of source control proved a strong, statistically significant predictor of mortality in nearly all subgroups of community-acquired, early- and late-onset hospital-acquired IAI, whether manifesting with localized or generalized peritonitis (Table 8). Mortality in patients with successful vs. failure of source control was significantly higher in pooled sepsis $(15.1 \%$ vs. $43.5 \%$; p $<0.001)$, septic shock $(27.0 \%$ vs. 58.0\%; $\mathrm{p}<0.001)$, combined sepsis and septic shock $(18.6 \%$ vs. $48.4 \% \%$; $<0.001)$. These data, although unadjusted, offer a more detailed explanation as to why in the total AbSeS database, containing 2621 critically ill patients with IAI, antimicrobial resistance was not recognized as an independent risk factor for mortality while failure of source control appeared pivotal.

The AbSeS study only focused on empiric antimicrobial therapy. However, the experience of the Merino trial suggests that definitive antibiotic therapy could also have its importance.[55] No study 
focusing on IAI ever compared the results of documented therapies against specific MDR microorganisms. In this context, we can imagine that results could differ from one drug to another based on unadjusted pharmacokinetic or minimal inhibitory concentration (MIC) criteria and that specific choices and dosing regimens may matter. To assess the impact of all these components of antimicrobial stewardship in the specific setting of truly complicated IAI would require even larger datasets that are not available and probably very difficult to achieve, without a coordinated effort.

\section{Limitations}

Limitations need to be acknowledged. This is an observational cohort study and therefore prone to bias. From some geographic regions only small numbers were available hampering meaningful conclusions. Because of the international scale and observational nature of the study no strict approach to source control was standardized. Also, evaluation of source control remained a subjective matter and because of the size of the study no independent panel for comprehensive evaluation of source control was established. Finally, no central lab for microbiology was organized. As such, we had to rely on the local labs for microbiological identification and susceptibility testing leading to an inherent variability in microbiological approach and, possibly, risk of misclassification.

\section{New insights, guidelines for empiric choices, and recommendations}

- In the face of rising antimicrobial resistance in key pathogens involved in IAI, we recommend to reach consensus through a collaborative effort of major scientific societies involved in surgery, intensive care and antimicrobial stewardship of these infections on the thresholds of resistance to redesign empiric therapy. In our opinion, within the context of IAI, different thresholds can be defined depending on the severity of disease expression, the challenge to achieve source control, and baseline rates of MDR in the index region. The same recommendation holds for a critical reassessment and stricter guidelines on the indications for microbiologic sampling, choices in directed therapy upon microbiological documentation, PK/PD optimization and duration of antimicrobial therapy.

- These recommendations probably need to be differentiated according to the previously proposed matrix grid including severity of disease presentation (the range from mild to severe disease with septic shock), degree of source control and late-onset of disease. In the latter, combined, in particular, with failure of source control, involvement of resistant pathogens, enterococci (including vancomycin-resistant E. faecium) and Candida species becomes more important. In this minority of cases combining late-onset hospital-acquired infection, diffuse 
peritonitis and septic shock, a restricted and specific setting, knowledge of local ecology and moreover of individual colonization status and information from relevant isolates from deep sampling becomes even more crucial to guide antimicrobial therapy. In the near future, the use of rapid diagnostic tests will reshape the decision-making process by a more timely selection of targeted antibiotic therapy.

- Once this consensus is established (and to a large extent reestablished as a number of principles have been previously corroborated), it will be key to translate these recommendations to the working field, in order to guarantee maximal penetration of antimicrobial stewardship. This will involve bottom up participation and motivation of physicians and care settings towards adherence and represents a greater challenge than the often-repeated publication of guidelines.

- Care bundles with checklist approaches, carried and mandated by individual institutions, may represent a future pathway towards achieving preset reasonable and achievable goals. As for any guideline, it will be an illusion to achieve full adherence, as guidelines will never be able to capture the full complexity of individual cases.

\section{Conclusion}

In distinct European regions, antimicrobial resistance is common in isolates from critically ill patients with IAI. Especially resistance against aminopenicillins, fluoroquinolones, and $3^{\text {rd }}$ generation cephalosporins in E. coli, K. pneumoniae and P. aeruginosa is problematic as is carbapenemresistance in the latter pathogen. Though resistance is an issue in every European region, substantial differences exist, stressing the need for steering antimicrobial therapy on basis of local resistance patterns, in the community and the hospital. In a subcohort of patients with secondary peritonitis and sepsis or septic shock the rate of empiric appropriate antimicrobial therapy was $64.8 \%$. Appropriateness of antimicrobial therapy did not impact on mortality. Source control, on the other hand, appeared to be a much more important player in this setting albeit that this concept remains difficult to objectivate. The current and worrisome resistance figures and the relative importance of antimicrobial therapy should incite scientific societies to redefine antimicrobial recommendations defining thresholds of acceptable resistance and considering local surveillance data. 


\section{DECLARATIONS SECTION}

\section{Conflicts of interest.}

$\mathrm{SB}$ received research grants from Pfizer.

PM served as speaker and advisory board member for Menarini, MSD and Pfizer.

Funding. AbSeS is a Trials Group Study of the European Society of Intensive Care Medicine. The study was supported by a Pfizer investigator-initiated research grant.

\section{ACKNOWLEDGEMENTS}

Collaborators AbSeS study: national coordinators and local investigators are listed in ESM-1. 